\title{
Distraction shrinks space
}

\author{
Jesse Q. Sargent • Jeffrey M. Zacks • \\ John W. Philbeck • Shaney Flores
}

Published online: 21 February 2013

(C) Psychonomic Society, Inc. 2013

\begin{abstract}
Research investigating how people remember the distance of paths they walk has shown two apparently conflicting effects of experience during encoding on subsequent distance judgments. By the feature accumulation effect, discrete path features such as turns, houses, or other landmarks cause an increase in remembered distance. By the distractor effect, performance of a concurrent task during path encoding causes a decrease in remembered distance. In this study, we ask the following: What are the conditions that determine whether the feature accumulation or the distractor effect dominates distortions of space? In two experiments, blindfolded participants were guided along two legs of a right triangle while reciting nonsense syllables. On some trials, one of the two legs contained features: horizontally mounted car antennas (gates) that bent out of the way as participants walked past. At the end of the second leg, participants either indicated the remembered path leg lengths using their hands in a ratio estimation task or attempted to walk, unguided, straight back to the beginning. In addition to response mode, visual access to the paths and time between encoding and response were manipulated to determine whether these factors would affect feature accumulation or distractor effects. Path legs with added features were remembered as shorter than those without, but this result was significant only in the haptic response mode data. This finding suggests that when people form spatial memory representations with the intention of navigating in room-
\end{abstract}

J. Q. Sargent $\cdot$ J. M. Zacks $\cdot$ S. Flores

Washington University in St. Louis, St. Louis, MO, USA

J. Q. Sargent $(\bowtie)$

Psychology Department, Francis Marion University, Florence, SC 29502, USA

e-mail: jsargent@fmarion.edu

J. W. Philbeck

George Washington University, Washington, DC, USA scale spaces, interfering with information accumulation substantially distorts spatial memory.

\section{Keywords Spatial cognition}

Knowledge of distances helps humans navigate, remain oriented, plan routes, and give directions. Attempts to measure mental representations of distance produce varying results depending, in large part, on how the distances are encoded and recalled (for reviews, see Montello, 1997, 2009). Previous studies have shown that across a range of circumstances, paths with more features (e.g., turns, intersections, landmarks) tend to be remembered as longer than paths with fewer features. In contrast, two studies by Glasauer and colleagues (Glasauer, Schneider, Grasso, \& Ivanenko, 2007; Glasauer et al., 2009) have shown that paths walked while counting backward by sevens are perceived as shorter than paths walked without a concurrent task. The present study examines the effects of path features on remembered distance for paths walked with a concurrent task. By using a conjunction of the conditions that give rise to the expanding and shrinking of distance representations, we aim to understand the mechanisms responsible for these contrasting, systematic distortions in spatial memory.

\section{Features make paths seem longer}

The bulk of the relevant research shows that paths associated with more features are remembered as longer than paths associated with fewer features (e.g., Allen, 1981; Allen \& Kirasic, 1985; Briggs, 1973; Jansen-Osmann \& Berendt, 2005). We will refer to this as the feature accumulation effect (Montello, 1997). For example, Sadalla and Staplin (1980a) required participants to follow experimental paths of masking tape, $8.53 \mathrm{~m}$ long, while wearing a blinder that restricted 
vision to approximately $0.91 \mathrm{~m}$ ahead. At the end of each experimental path, participants walked a short $(1.52-\mathrm{m})$ reference path and then drew the relative length of the experimental path on a piece of paper that showed a representation of the reference path (ratio estimation). Distance estimates were longer for paths that contained more intersections (other, crossing masking tape paths). Sadalla and colleagues also showed that paths on which the masking tape intersections were labeled with high-frequency names were judged to be longer than equivalent length paths marked with lowfrequency names (Sadalla \& Staplin, 1980b; Sadalla, Staplin, $\&$ Burroughs, 1979). These data suggested that the observed feature accumulation effect was driven by participants' greater ability to recall the high-frequency names, which resulted in the association of the high-frequency-name paths with more information. The authors interpreted these results as consistent with an information storage account of the feature accumulation effect; remembered route length scales with the amount of information, spatial or otherwise, associated with that route in memory.

Hutcheson and Wedell (2009) provided a theoretical explanation of the feature accumulation effect, based on dual memory systems, that refines the information storage account. They showed that the feature accumulation effect increases when a concurrent task is included during path encoding or when a distractor task is included during a delay period between encoding and responding. The authors noted that this increase in the feature accumulation effect with decreasing memory fidelity contrasts with the results of Sadalla and Staplin (1980b). Sadalla and Staplin (1980b) showed that the feature accumulation effect was greater when more information about the route (names that labeled the intersections) was available in memory. Hutcheson and Wedell explained this discrepancy in terms of a dual memory systems account. This view distinguishes between fine-grained, metric spatial memory, which is more precise but harder to encode/maintain, and categorical or nonmetric spatial memory, which is less accurate but more robust (Huttenlocher, Hedges, \& Duncan, 1991). According to this account, the feature accumulation effect results from the influence of nonmetric memory codes, which increases when more fragile metric spatial codes are less available. Sadalla and Staplin's (1980b) name frequency manipulation served to increase the relative availability of nonmetric codes (how many names are associated with a given path) and, thus, increased the feature accumulation effect. Hutcheson and Wedell employed manipulations (concurrent tasks and filled delays) that served to decrease the relative availability of metric spatial codes, which also increased the feature accumulation effect (see also JansenOsmann \& Wiedenbauer, 2006). Thus, the dual memory systems account suggests that the feature accumulation effect depends not only on the quantity of information associated with a route, but also on the quality of that information as well.
The feature accumulation effect has been demonstrated in several studies comparing remembered distances for paths containing a greater and smaller number of turns. For example, Sadalla and Magel (1980, Experiment 1) required participants to first walk two 60.96-m experimental paths, one containing two turns, the other containing seven turns, and then to walk a straight $30.48-\mathrm{m}$ reference path. The remembered distances of the experimental paths were then indicated by drawing a vertical hash mark on a horizontal response line that had the length of the reference path already marked on it (ratio estimation). The seven-turn paths were remembered as significantly longer than the two-turn paths. This variety of feature accumulation effect, termed the angularity effect, has been replicated using different numbers of turns and different behavioral measures of path length (e.g., reproduction of walking distances) (Sadalla \& Magel, 1980, Experiments 2 and 3) and in virtual reality (Hutcheson \& Wedell, 2009; Jansen-Osmann \& Berendt, 2002).

Consistent with a dual memory systems account of feature accumulation effects, the most consistent moderator of the angularity effect appears to be the degree to which memory for the routes is taxed during either encoding or maintenance (e.g., Hutcheson \& Wedell, 2009). Because metric representations are less durable, conditions that challenge memory systems are likely to result in relative reliance on nonmetric memory codes. For example, Jansen-Osmann and Wiedenbauer (2006) observed an angularity effect when distance estimates were made after walking all paths (two turn, seven turn, reference), but no effect when estimates were made after each path. As participants walk and encode additional path lengths, the relative availability of fine-grained, metric spatial information decreases, and the influence of nonmetric information (number of turns) emerges. Likewise, angularity effects may be elusive in prospective distance judgments because foreknowledge of the upcoming spatial task results in particular effort directed toward encoding and maintaining metric information.

Another perspective that has been proposed to explain feature accumulation effects focuses on the role of features as segmenters of associated routes. Route segmentation accounts suggest that path features break routes up into subsections and that routes associated with more sections are remembered as being longer (Allen, 1981; Allen \& Kirasic, 1985; Jansen-Osmann \& Berendt, 2005; Montello, 1997). The tendency for human memory systems to segment space into manageable chunks is well documented (e.g., Gobet et al., 2001; Sargent, Dopkins, Philbeck, \& Chichka, 2010; Wang \& Brockmole, 2003). In addition, there is evidence that distances between locations in different chunks are exaggerated in spatial memory, relative to distances between locations in common chunks (Allen \& Kirasic, 1985; McNamara, Hardy, \& Hirtle, 1989; for a discussion of possible mechanisms, see 
Holyoak \& Mah, 1982; Sadalla \& Magel, 1980; Zacks, Speer, Swallow, Braver, \& Reynolds, 2007). Unlike the information storage and dual memory systems accounts, the route segmentation account predicts that the feature accumulation effect depends on the ability of features to segment the route. Thus, features that are homogeneous and continuous throughout a route should be less likely to produce the feature accumulation effect than should features that cause a route to be segmented into chunks.

\section{Concurrent tasks make paths seem shorter}

Evidence that concurrent tasks can actually cause a decrease in distance estimates for associated paths comes from studies of self-motion reproduction (Glasauer et al., 2007; Glasauer et al., 2009). In the most relevant experiments, blindfolded participants walked along outbound paths, between 9.14 and $18.29 \mathrm{~m}$ in length, and then reproduced the paths either by continuing on in the same direction as the outbound path or by turning around and walking back to the origin. When participants were required to perform a concurrent mental task (counting backward by sevens) during only the outbound path, subsequent reproductions were shorter. Conversely, when participants were required to perform the concurrent task during only the reproductions, those reproductions were longer. In other words, performing a concurrent task while walking a path led to a shorter estimate of that path's distance. Glasauer and colleagues suggested that counting backward by sevens distracted participants from the accumulation of temporal or distance cues (or both), resulting in shorter distance representations. Following Glasauer, we will call this the distractor effect. An analogous mechanism has been proposed by researchers studying temporal duration judgments. The attentional allocation model posits that if more attention is devoted to the perception of passing time, subsequent judgments of duration will be longer because increased attentional resources facilitate the accumulation of temporal cues ${ }^{1}$ (e.g., Brown, 1997; Brown \& West, 1990; Hicks, Miller, Gaes, \& Bierman, 1977; Zakay, 1989).

While counting backward by sevens might not be a path feature per se, it might still result in a path being associated with a greater amount of information. So why did Glasauer et al. (2007; Glasauer et al., 2009) find that concurrent tasks consistently decrease remembered distances? Likewise, why do the various path features described in literature reporting

\footnotetext{
${ }^{1}$ Because the effect of distraction on duration perception is well documented, distorted temporal representations are likely to play a role in how distraction affects distance perception. However, neither the present data nor those of Glasauer and colleagues (2007; Glasauer et al., 2009) specifically indicate that time perception is involved in the observed effects of distraction on distance knowledge.
}

the feature accumulation effect show no evidence of distracting participants from the accumulation of distance cues? One possibility is that in the paradigm used by Glasauer and colleagues, the concurrent task was simply more distracting than the path features that lead to the feature accumulation effect. Another possibility is that the feature accumulation effect depends on the segmentation of paths into multiple discrete parts and concurrent tasks do not segment paths as well as features do. The contrasting effects might also have resulted from procedural differences-for example, in the degree of visual access at encoding and in the method of assessing distance knowledge. The present study evaluates these possibilities.

\section{Present study}

In order to understand the conditions under which the distractor and feature accumulation effects obtain, we examined four potentially critical differences between the paradigms that have found feature accumulation effects and those that have found distractor effects. First, in studies showing the feature accumulation effect, the features were punctate and environmental. That is, the features were of limited spatial and temporal extent relative to the entire path, and they were part of the physical environment. These landmark characteristics were not shared by the concurrent tasks that caused the distractor effect. The present study tested for the distractor effect using punctate environmental features. One possibility is that the perception of these features requires less mental effort than does counting backward by sevens and, thus, is less distracting. The punctate environmental features used in this study (gates) were linked to changes in a concurrent task (recitation of a nonsense syllable); a new syllable was given at each gate. So in addition to being generally distracting throughout the paths, this concurrent task demanded increased attention precisely at time points when punctate environmental features also demanded attention. Thus, if taxing attentional resources by distraction underlies the distractor effect, the inclusion of this linked concurrent task should increase the relative probability that punctate environmental features will cause the distractor effect, rather than the feature accumulation effect.

However, requiring a change in the concurrent task at each punctate environmental feature may also serve to increase the salience or memorability of those features and, thus, increase the likelihood of the feature accumulation effect (Hutcheson \& Wedell, 2009; Sadalla \& Staplin, 1980b). Furthermore, the association of each path segment with different concurrent task features (syllables) might distinguish those segments in memory. This also might be expected to increase the likelihood of the feature accumulation effect. It has been suggested 
that the feature accumulation effect arises because features segment associated routes into discrete parts and routes remembered as having more parts are remembered as being longer (e.g., Allen \& Kirasic, 1985; Jansen-Osmann \& Berendt, 2005). The concurrent tasks used by Glasauer and colleagues may fail to make paths seem longer because they do not segment those paths. We tested this possibility by looking at the effect of punctate features on remembered path length.

A second difference between paradigms showing distractor and feature accumulation effects is that demonstrations of the feature accumulation effect involve at least some visual access to the environment, whereas the distractor effect has been observed only in blindfolded participants. One might expect path features to be considerably less distracting when paths are encoded with visual input. For example, vision provides a preview of upcoming features so that they may be less surprising and, thus, less distracting. Experiment 2 asks whether the distractor effect can be obtained even when visual information is available at encoding.

Third, the feature accumulation effect increases when a filled delay occurs between encoding and responding (Hutcheson \& Wedell, 2009), but the distractor effect has been observed only when encoding immediately precedes responding. There is support, reviewed above, for the idea that the feature accumulation effect arises from the influence of nonmetric spatial information on distance estimation (e.g., paths with more segments seem longer, regardless of the metric properties of the segments). Relative reliance on nonmetric spatial properties increases as the availability of less durable metric information decreases. Therefore, inserting a filled delay between encoding and response may serve to increase the relative influence of the feature accumulation effect. Experiment 2 tested this possibility as well.

Fourth, whereas the feature accumulation effect has been observed using a number of response modes, including psychophysical scaling techniques such as ratio estimation and action-based methods such as distance reproduction (Sadalla \& Magel, 1980), the distractor effect has been observed only in reproduction tasks. Glasauer and colleagues suggested that the distractor effect, which is well established in time perception tasks, influences the representations of time used for movement reproduction. It has also been suggested that movement reproduction tasks depend not on static distance estimates but, rather, on the storage of velocity profiles that can be recreated at response (Berthoz, Israel, Georges-Francois, Grasso, \& Tsuzuku, 1995). If the distractor effect operates strictly by influencing the temporal aspects of an experienced (to be reproduced) velocity profile, we would expect no distractor effect in responses that do not depend on a stored velocity profile. We tested this possibility by looking for the distractor effect in ratio estimation and triangle completion tasks, neither of which can be performed by reproducing movements or durations.

In sum, in this study, we asked four questions about the effects of distraction on spatial encoding: Can we find evidence that features distract from the accumulation of distance cues when (1) those features are physical and punctate (as opposed to continuous), (2) participants have visual access to the environment, (3) there is a filled delay between encoding and responding, and (4) distance estimates are made by ratio estimation and triangle completion? We used a paradigm in which participants walked short L-shaped paths ( $L$ paths) with limited or no vision and then either estimated the lengths of the path legs using their hands or walked, unguided, back to the beginning of the path (triangle completion).

\section{Experiment 1}

In Experiment 1, we asked whether continuous, nonphysical distractors and action-based responding are necessary conditions for the distractor effect by looking for the effect with punctate environmental features in ratio estimation responding. Blindfolded participants walked a series of $\mathrm{L}$ paths while reciting a nonsense syllable and, at the end of each $\mathrm{L}$ path, either used their hands to estimate the lengths of the two path legs by ratio estimation (explicit, haptic response mode) or performed a triangle completion task (action-based, walking response mode). Analyses tested for the distractor effect using discrete path features and two different response modes.

\section{Method}

\section{Participants}

Twenty-five undergraduates (16 females) participated in this study in exchange for course credit. Their mean age was 20 years (range: $18-28$ ).

\section{Apparatus}

The experiment took place inside a large room $(8.23 \times$ $15.54 \mathrm{~m}$ ) in which two identical L paths were set up. Starting and stopping points for leg 1 and leg 2, respectively, were marked on the paths for the three levels of path leg length manipulation (leg 1/leg 2): long $(6.10 \mathrm{~m}) / \operatorname{long}$ $(6.10 \mathrm{~m})$, short $(4.88 \mathrm{~m}) /$ long $(6.10 \mathrm{~m})$, and long $(6.10 \mathrm{~m}) /$ short $(4.27 \mathrm{~m})$. These are illustrated in Fig. 1 . The short version of leg 2 was slightly shorter than the short version of leg 1 in order to discourage participants from representing the path lengths nonmetrically, dichotomously, as either long or short. Participants alternated between the 


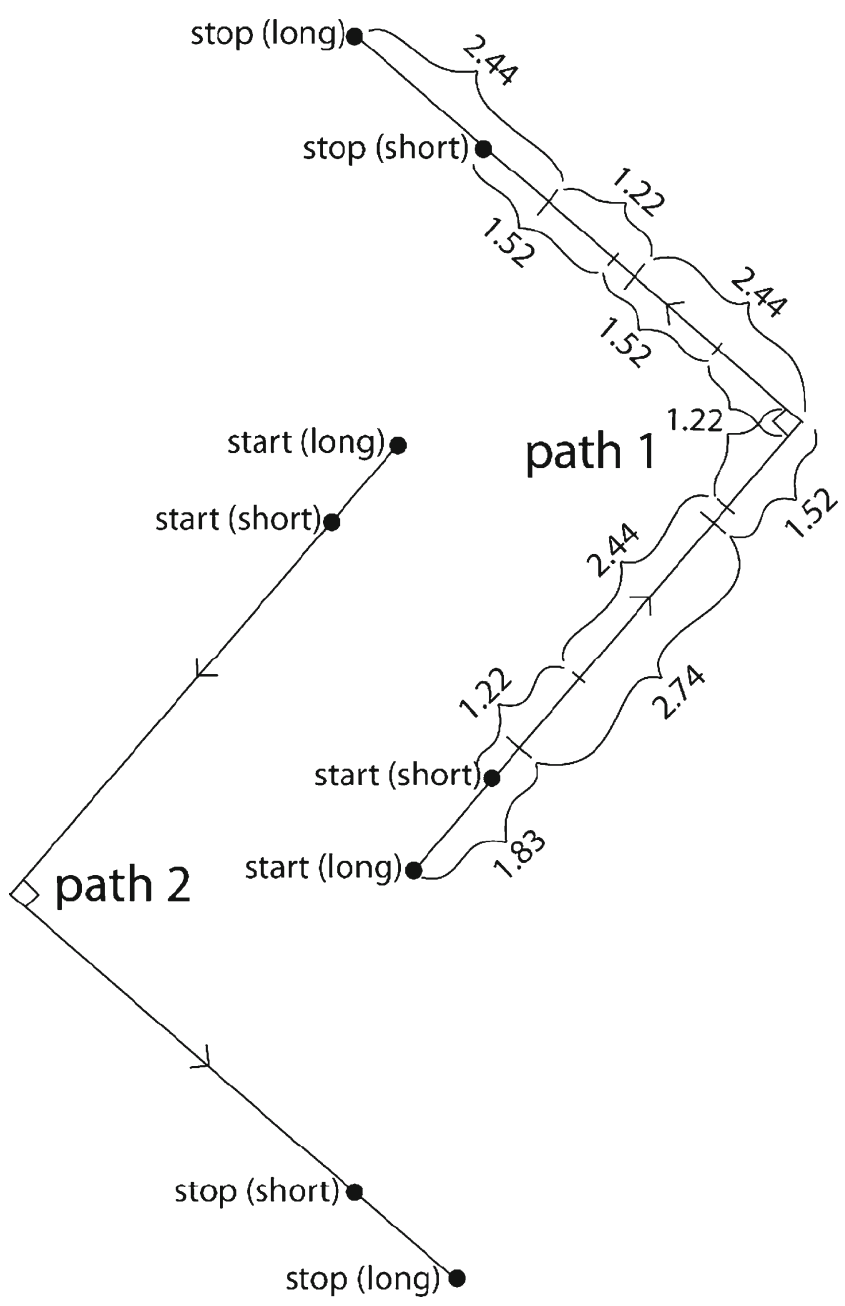

Fig. 1 Overhead view scale diagram of L paths showing locations of starting and stopping points and path features. Longer hash marks on path legs are locations of features for long-path conditions; shorter hash marks are locations of features for short-path conditions. Distances are in meters

two L paths on successive trials to avoid error feedback on walking trials. The path features were gates through which blindfolded participants were led; they were made from flexible car antennas mounted horizontally on movable stands. The antennas bent out of the way on participants' torsos as they walked past. For trials on which the $\mathrm{L}$ paths contained features, the gates were on either leg 1 or leg 2 , never both. Legs with features always contained two gates. The gate locations on the paths, by leg length condition, are shown in Fig. 1. Hearing protectors provided $29 \mathrm{~dB}$ of noise reduction, and blindfolds were of the soft light-blocking variety designed for sleeping. Experimenters led participants using a dowel $0.61 \mathrm{~m}$ long and $2.54 \mathrm{~cm}$ in diameter. The experimenter held the middle of the dowel horizontally, at chest level, in front of the participants, who grabbed the ends of the dowel, one in each hand.

\section{Procedure}

Participants were met outside the experimental room, where they were briefed and gave consent to participate. After receiving instructions, they were fitted with a blindfold and hearing protectors and led into the experimental room. Participants were told that they would be led along paths of varying lengths and sometimes they would be asked to show the lengths of the paths using their hands; other times, they would be asked to walk back to the start of the paths on their own. Participants were also instructed that as they were led along the various experimental paths, they would recite, out loud, a nonsense syllable (e.g., val, jub, niv, ver), which would be called out by an experimenter. Different nonsense syllables were randomly assigned to the different $\mathrm{L}$ path conditions. Participants were instructed not to synchronize the syllables with their steps and, generally, not to count anything throughout the experiment in order to discourage the use of counting as a basis for subsequent distance estimates. Also, participants were warned that they would occasionally pass through a gate and that a new nonsense syllable would be given at each gate and at the $90^{\circ}$ turns on the L paths. So path features were not only the gates, but also contemporaneous changes in the nonsense syllable being recited.

First, participants were led along a straight comparator path $(12.19 \mathrm{~m})$. At the end of this path, their hands were placed on a yard stick mounted horizontally on a stand at waist level, with a rubber band marking a distance $68.58 \mathrm{~cm}$ from the left end of the stick. They were told that the distance from the end of the stick to the rubber band represented the length of the comparator path and that they would be asked to estimate with their fingers the lengths of other paths on the measuring stick relative to the comparator path. The comparator path was then walked a second and final time to provide a better sense of the comparator distance.

Then two practice trials walking the $\mathrm{L}$ paths were completed, one haptic and one walking response, to make sure participants were comfortable with the tasks. For haptic response trials, participants indicated on the yardstick, separately, the lengths of the first and second legs of the L path they had just walked, relative to the comparator path. For walking response trials, participants were instructed to turn left and walk straight back to the origin. The stopping place was then recorded, using reel measuring tapes, as the distance from each of two anchor points. Each anchor point was a bolt protruding through a piece of sheet metal that was taped to the floor, one on each side of the experimental room. The ends of the reel tape measures were hooked over the bolts. Response times were recorded in seconds. Times to walk leg 1 and leg 2 and to complete the response were all recorded separately for each trial. The experimenter guiding participants along the $\mathrm{L}$ paths (the same experimenter guided all participants) tried to achieve a plateau-shaped velocity 


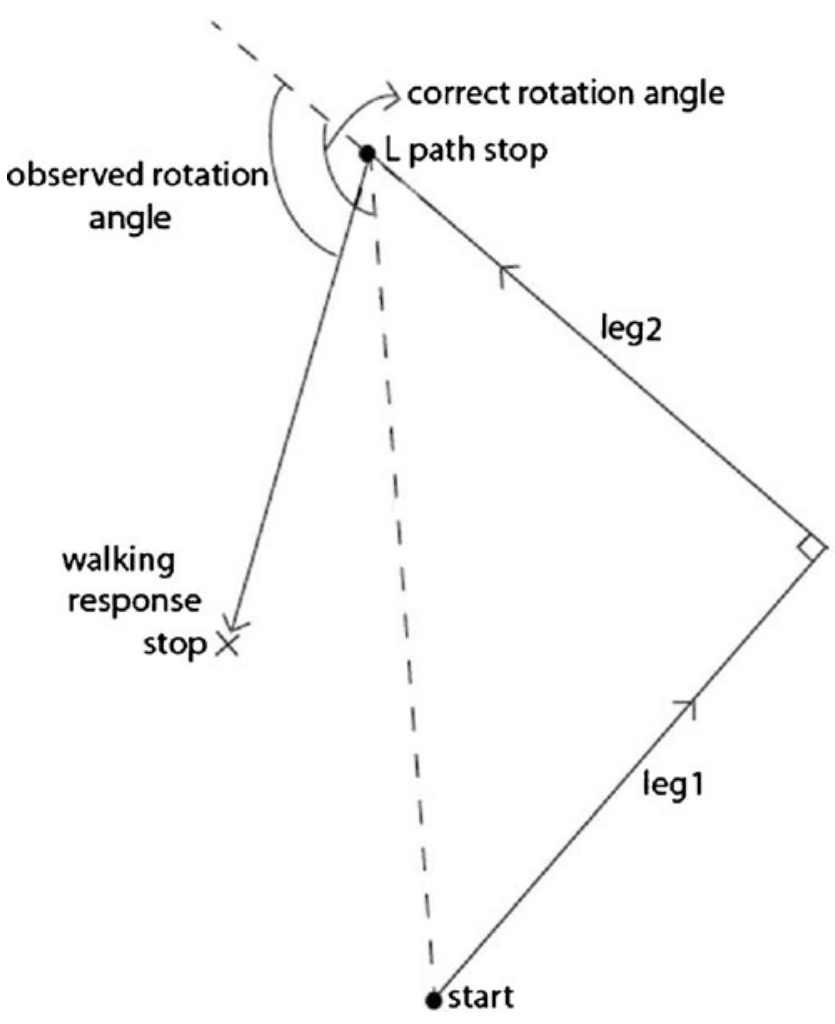

Fig. 2 Observed rotation angle

profile that might be considered typical for walking with vision. Maximum velocity was also typical for walking with vision but was determined, to some extent, by what individual participants were comfortable with. Experimental sessions lasted approximately $60 \mathrm{~min}$.

\section{Design}

A within-subjects design was used: 2 (response mode: haptic vs. walking) $\times 3$ (features: gates on leg 1 or leg 2 or no gates) $\times 3$ (leg length: long/long, long/short, short/long). ${ }^{2}$ Each participant completed 30 trials. One trial was run for each of the 18 conditions, except the long/long conditions; these were run 3 times each in order to examine effects of response mode and features on response variability. Order of the trial conditions was randomized separately for each participant.

\section{Results}

For haptic responses, the raw data were the leg lengths indicated by participants on the yard stick. For walking responses, the raw data were the angles through which participants rotated, counterclockwise, at the ends of the $\mathrm{L}$

\footnotetext{
${ }^{2}$ For ease of interpretation and because the leg length manipulation was not of primary interest, in the statistical analyses, response data were collapsed across the two shorter leg lengths $(4.27$ and $4.88 \mathrm{~m})$ to create two categorical levels of this variable, short and long.
}

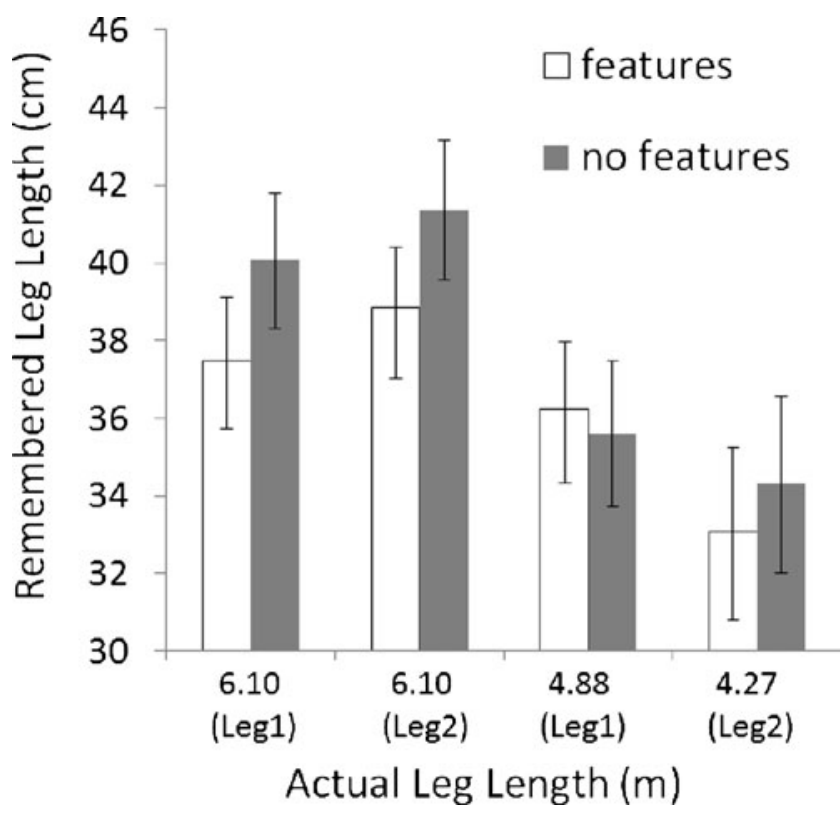

Fig. 3 Haptic, ratio estimation data for Experiment 1 showing remembered leg length as a function of path features, actual leg length, and leg order. Error bars show the standard errors of the means (Correct responses based on relative length of comparator path $(12.19 \mathrm{~m})$ and corresponding haptic response distance $(68.58 \mathrm{~cm})$ are $29.6,23.7$, and $20.74 \mathrm{~cm}$ for the $6.10-, 4.88-$, and $4.27-\mathrm{m}$ paths, respectively. Thus, in absolute terms, paths lengths were grossly overestimated in the haptic response data. However, ratio estimation ability per se was not a focus in the present study. For example, participants underwent only two trials on which the relationship between walked and haptic response distances was demonstrated at the outset of the experimental session. Therefore, we do not discuss this result)

paths as indicated by the stopping locations of participants on unguided paths (observed rotation angle in Fig. 2).

We first present results for the haptic responses (see Fig. 3). A three-way, 2 (features, no features) $\times 2$ (long [6.1 m], short [4.3-4.9 m]) $\times 2$ (leg 1, leg 2) ANOVA showed a main effect of features: Paths with features were remembered as being shorter than those without, $F(1,24)=4.33, p=.048$. A main effect of leg length was also observed, $F(2,24)=63.40, p<.001$. No other main effects or interactions reached significance. The effect of leg length shows that our behavioral measures were sensitive to actual differences in leg length. The effect of features indicates that paths with gates and syllable changes were remembered as being shorter than those without. Thus, the distractor effect, previously observed for continuous (concurrent task) distractors in motor reproduction response data, was demonstrated using discrete, physical distractors in ratio estimation response data.

In the walking response mode, independent data for each individual path leg were not available. Therefore, walking data are presented by $\mathrm{L}$ path condition, rather than by $\mathrm{L}$ path leg condition (see Fig. 4). Greater observed rotation values indicate greater remembered length for leg 2 relative to leg 1 
(see Fig. 2). A two-way, 3 (features on leg 1, no features, features on leg 2) $\times 3$ (short/long, long/long, long/short) ANOVA showed a main effect of relative leg length, $F(2$, $48)=12.69, p<.001$. No other effects reached significance. As with the haptic data, the walking response data showed sensitivity to actual differences in leg length; participants rotated more as the relative length of leg 2 to leg 1 increased. However, there was no significant main effect of features in the walking data, nor did any simple main effects of features at any of the levels of leg length reach significance.

Figure 4 shows a general tendency to under-rotate. Although participants were explicitly told that the turn between legs 1 and 2 was always 90 , rotation error may have been influenced by misperception of this turn or by bias in the response turn. Previous work using triangle completion tasks has shown that people show rotation bias toward preestablished, internal default values such that larger turns tend to be underestimated and smaller turns tend to be overestimated (Fujita, Klatzky, Loomis, \& Golledge, 1993; Klatzky, Beall, Loomis, Golledge, \& Philbeck, 1999; Loomis et al., 1993). In the present experiment, because the turn between the two path legs was always $90^{\circ}$, and because this is a canonical turn size, it is possible that $90^{\circ}$ was a common default value. Given that the correct response rotation angles were all greater than $90^{\circ}$, the observed under-rotation may reflect a bias toward the canonical $90^{\circ}$ angle. In any case, we do not suggest that rotation

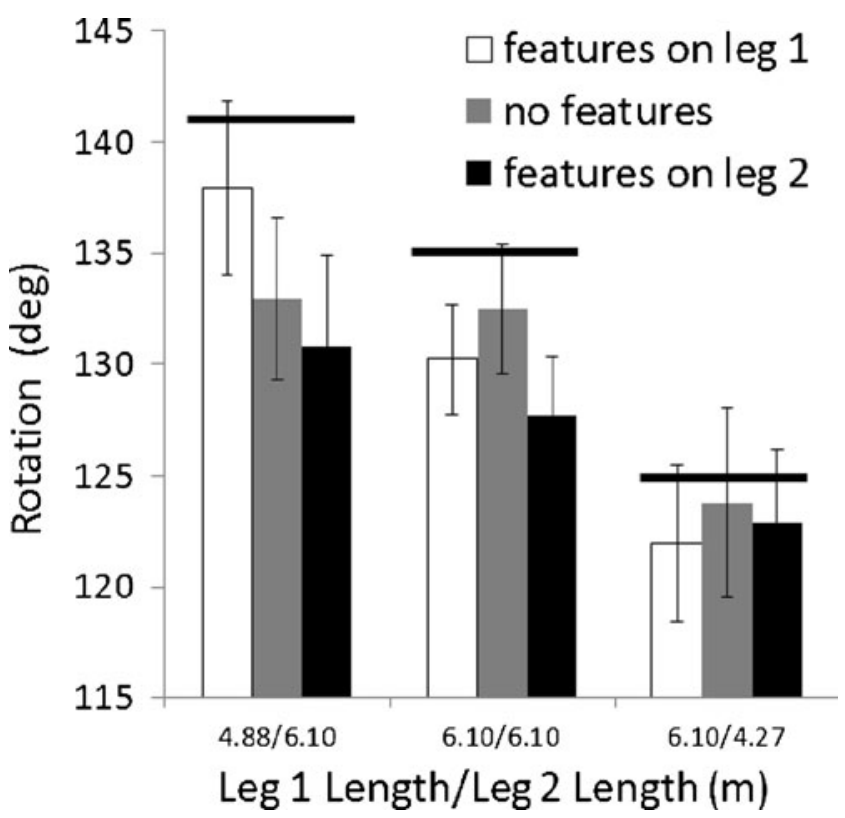

Fig. 4 Walking response data for Experiment 1 showing degree of counterclockwise rotation exhibited by participants at the end of leg 2 as a function of feature placement and relative leg lengths. Greater values indicate greater remembered length for leg 2 relative to leg 1 . Bold lines show correct rotations for each condition. Error bars show the standard errors of the means error reflects an unbiased measure of remembered leg lengths but, rather, assume that any bias is independent of the effect of features, our variable of primary interest.

Finally, we examined the time taken to walk the paths. Repeated measures ANOVAs showed a significant main effect of features on walk time, $F(1,24)=7.71, p=.01$ (see Table 1). Despite efforts to keep velocity across all paths consistent, paths with features took slightly longer than paths without. Possible confounding of the effect of features with the effect of path duration is of little concern in the haptic response data because paths with features, although associated with slightly longer durations, were remembered as shorter in length. To test whether controlling for walk time might reveal a significant effect of features in the walking response data, for each participant, we first regressed rotation angle (see Fig. 2) on the ratio of walk times for the two path legs (leg 1 time/leg 2 time). Then we repeated the ANOVA described above using the residuals from these regressions, instead of rotation angle, as the dependent variable. The effect of features still did not approach significance. The walk time data also showed that time from stopping at end of leg 2 to completion of responses was greater for haptic than for walking responses and that time to complete walking responses was slightly longer for trials on which either path leg contained features, $t(24)=2.10, p=.047$ (see Table 2).

\section{Discussion}

In Experiment 1, we found a distractor effect in the ratio estimation haptic task, but not in the triangle completion walking task. In the haptic data, the distractor effect emerged even under conditions that might have been expected to produce the opposite result. First, punctate features caused paths to be remembered as shorter. This suggests that distractors need not be continuous throughout the paths in order to distract from the accumulation of distance cues. Even discrete features that segment paths into multiple parts can lead to shortening of remembered path lengths. Second, the distractor effect was found in a ratio estimation task in which distance representations must be rescaled and indicated haptically. This suggests that if the

Table 1 Mean times (in seconds) to walk paths (and SDs across participants) by leg length and feature (gates/no-gates) conditions, for Experiments 1 and 2

\begin{tabular}{|c|c|c|c|c|c|c|}
\hline \multirow{2}{*}{$\begin{array}{l}\text { Leg length: } \\
\text { Features: }\end{array}$} & \multicolumn{2}{|l|}{$6.10 \mathrm{~m}$} & \multicolumn{2}{|l|}{$4.88 \mathrm{~m}$} & \multicolumn{2}{|l|}{$4.27 \mathrm{~m}$} \\
\hline & Gates & $\begin{array}{l}\text { No } \\
\text { gates }\end{array}$ & Gates & $\begin{array}{l}\text { No } \\
\text { gates }\end{array}$ & Gates & $\begin{array}{l}\text { No } \\
\text { gates }\end{array}$ \\
\hline $\begin{array}{l}\text { Experiment } \\
1\end{array}$ & $\begin{array}{l}8.17 \\
(.17)\end{array}$ & $\begin{array}{l}7.81 \\
(.16)\end{array}$ & $\begin{array}{l}6.78 \\
(.19)\end{array}$ & $\begin{array}{l}6.67 \\
(.14)\end{array}$ & $\begin{array}{l}6.36 \\
(.20)\end{array}$ & $\begin{array}{l}6.14 \\
(.15)\end{array}$ \\
\hline $\begin{array}{l}\text { Experiment } \\
2\end{array}$ & $\begin{array}{l}6.91 \\
(.14)\end{array}$ & $\begin{array}{l}6.73 \\
(.14)\end{array}$ & $\begin{array}{l}5.81 \\
(.15)\end{array}$ & $\begin{array}{l}5.83 \\
(.14)\end{array}$ & $\begin{array}{l}5.33 \\
(.14)\end{array}$ & $\begin{array}{l}5.03 \\
(.11)\end{array}$ \\
\hline
\end{tabular}


Table 2 Mean times (in seconds) from stopping at end of leg 2 until completion of haptic or walking responses (and SDs across participants) by feature (gates/no-gates) conditions, for Experiments 1 and 2

\begin{tabular}{|c|c|c|c|c|}
\hline \multirow{2}{*}{$\begin{array}{l}\text { Response } \\
\text { mode: } \\
\text { Features: }\end{array}$} & \multicolumn{2}{|l|}{ Haptic } & \multicolumn{2}{|l|}{ Walking } \\
\hline & Gates & No gates & Gates & No gates \\
\hline Experiment 1 & $\begin{array}{l}19.38 \\
(0.76)\end{array}$ & $\begin{array}{l}19.34 \\
(0.97)\end{array}$ & $\begin{array}{l}17.73 \\
(0.67)\end{array}$ & $\begin{array}{l}17.00 \\
(0.60)\end{array}$ \\
\hline Experiment 2 & $\begin{array}{c}42.19 \\
(1.61)\end{array}$ & $\begin{array}{c}41.93 \\
(1.80)\end{array}$ & $\begin{array}{c}42.64 \\
(1.18)\end{array}$ & $\begin{array}{c}42.14 \\
(1.29)\end{array}$ \\
\hline
\end{tabular}

distractor effect operates by warping temporal representations, as suggested by Glasauer et al. (2007; Glasauer et al., 2009), these representations are shared between systems that guide motor reproduction and ratio estimation.

By the same logic, the absence of a distractor effect in the walking response mode data might indicate that warped temporal representations do not play as large a role in triangle completion tasks. Motor reproduction may be performed by reproducing stored temporal profiles (e.g., Berthoz et al., 1995), and ratio estimation may be performed by simply reporting remembered path durations in spatial units. However, triangle completion tasks are more likely to depend on the construction of flexible environmental representations, which may be less influenced by temporal biases. We revisit the null result in the walking response data in the discussion of Experiment 2.

\section{Experiment 2}

In Experiment 2, we asked whether either visual deprivation or immediate responding is a necessary condition for the distractor effect. Previously, the distractor effect has been observed only in blindfolded participants, responding immediately after path encoding (Glasauer et al., 2007; Glasauer et al., 2009). Either or both of these procedural features might be critical for punctate features to cause the distractor effect. For example, one might expect path features to be less distracting when participants can see them coming. Also, filled delays have been shown to increase the feature accumulation effect (Hutcheson \& Wedell, 2009). Experiment 2 attempted to replicate the distractor effect using multiple response modes (as in Experiment 1), visual access to the paths at encoding, and a filled delay between path encoding and responding.

Method

\section{Participants}

Twenty-five Washington University undergraduates (12 females) participated for course credit. One participant did not complete the experimental session due to mild disorientation and dizziness. The mean age of the group was 19 years (range: 18-26 years).

\section{Materials}

The following changes were made to the materials used in Experiment 1. The hearing protectors and blindfold were replaced with earplugs and a welder's helmet, with a blackout curtain attached to both the front and back of the helmet. The flap of curtain on the front of the helmet could be taped up, allowing participants to see under the welding visor approximately $1.22 \mathrm{~m}$ in front of them and $0.61 \mathrm{~m}$ to either side. The paths were marked by colored string running along the floor that participants could see and follow when the flap was up.

The Brooks (1968) letter task was used as a distractor task to be performed between path encoding and response. This visual imagery task requires participants to picture a given block letter and trace clockwise around the outside of the image in the mind's eye, classifying each corner as being on the extreme top or bottom of the letter, for which a verbal "yes" response is given, or anywhere else, for which a verbal "no" response is given. Hutcheson and Wedell (2009) used a Shepard-Metzler mental rotation task as a distractor during the delay between encoding and response. We used the Brooks letter task because it is a spatial mental imagery task that can be administered to blindfolded participants.

\section{Procedure}

The following changes were made to the procedure from Experiment 1. Before entering the experimental room, participants were instructed that they would be allowed to see about $4 \mathrm{ft}$ in front of them while being led along the L-shaped paths but, otherwise, vision would be occluded throughout the experiment. Also, participants practiced performing the distractor (Brooks letter) task. At the beginning of each L-shaped path, the flap on the helmet was raised so that participants had limited vision as they were led along the $L$ paths. At the end of each $L$ path, before haptic or walking responses were given, the flap on the helmet was lowered, and participants performed the distractor task for $30 \mathrm{~s}$ on three letters $(10 \mathrm{~s}$ each $)$ as they were called out by an experimenter. Experimental sessions lasted approximately $90 \mathrm{~min}$.

\section{Results}

Data were analyzed as in Experiment 1. Results for the haptic response data were similar to those observed in Experiment 1 (see Fig. 5). A repeated measures ANOVA showed main effects of features, $F(1,23)=10.03, p=.004$, and leg length, $F(2,23)=50.85, p<.001$. No other effects reached significance. Again, participants showed sensitivity 


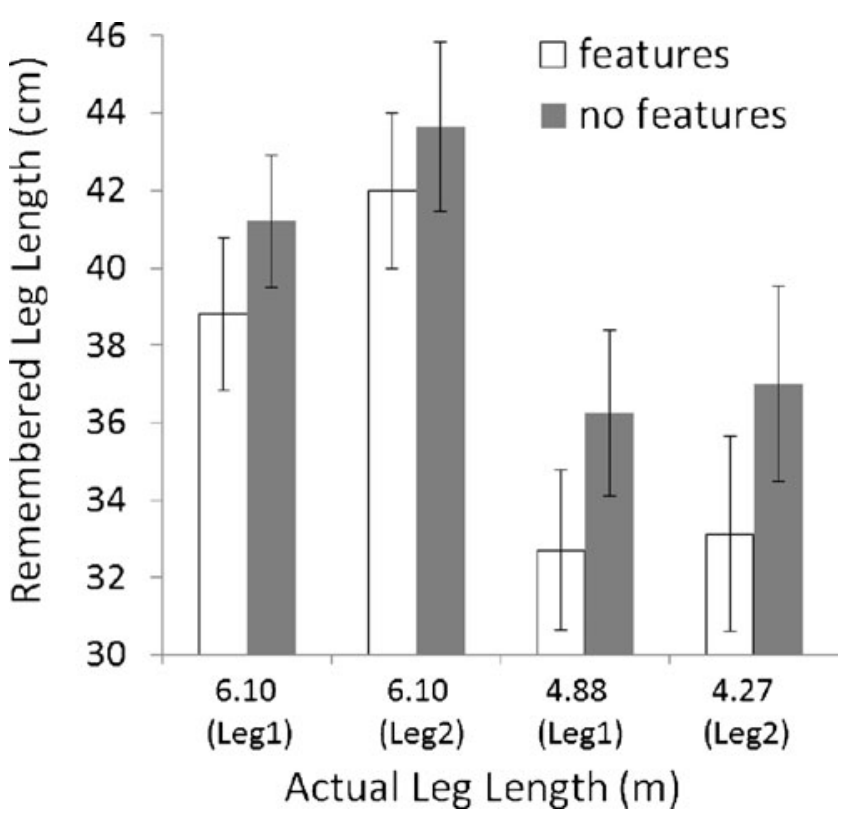

Fig. 5 Haptic, ratio estimation data for Experiment 2 showing remembered leg length as a function of path features, actual leg length, and leg order. Error bars show standard errors of the means

to actual path length, and more important, paths with features were remembered as being shorter than those without.

The results for the walking response mode were also similar to those in Experiment 1. A repeated measures ANOVA showed a main effect of relative leg length, $F(2,46)=18.22, p<.001$, but no other effects reached significance (see Fig. 6). The walking response data again showed sensitivity to actual differences in leg length but no effect of features. Figure 6 shows the tendency, observed in Experiment 1, to under-rotate, except when the correct rotation was smallest, when the second leg was short. This pattern is consistent with previous triangle completion studies showing that participants under-rotate when larger turns are in order and over-rotate for smaller turns (Fujita et al., 1993; Klatzky et al., 1999; Loomis et al., 1993).

Tables 1 and 2 show that the pattern of temporal data for Experiment 2 is similar to that for Experiment 1. Repeated measures ANOVAs showed a significant main effect of features on walk time, $F(1,23)=8.67, p=.007$; paths with features took slightly longer than paths without (see Table 1). We reran the analysis of walking response mode data controlling for walk time, as described in Experiment 1. Again, this analysis failed to reveal a significant effect of features. No significant differences were seen in response times (see Table 2). Walk times were markedly shorter in these data than in Experiment 1, probably reflecting a tendency among participants to walk faster when allowed some visual access to the environment. As compared with Experiment 1, response times are much longer and more variable in Experiment 2 (see Table 2) because they include the filled delay.

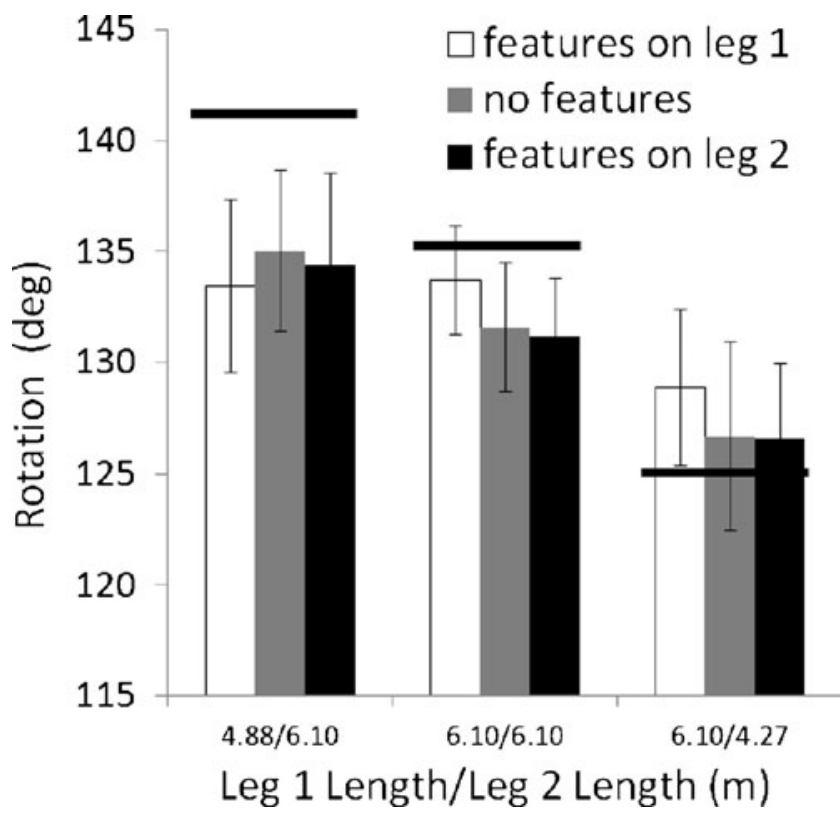

Fig. 6 Walking response data for Experiment 2 showing degree of counterclockwise rotation exhibited by participants at the end of leg 2 as a function of feature placement and relative leg lengths. Greater values indicate greater remembered length for leg 2 relative to leg 1 . Bold lines show correct rotations for corresponding conditions. Error bars show standard errors of the means

\section{Discussion}

The results of Experiment 2 again show a distractor effect, but only in the ratio estimation task. Even when distances are encoded with vision and there is a filled delay between encoding and response, the presence of additional path features at encoding results in a reduction in remembered path lengths as measured by ratio estimation. The conditions under which the distractor effect obtains are further extended here to conditions previously associated with the contrasting feature accumulation effect.

Walking response mode data again suggest that the mechanisms by which the distractor effect occurs play a smaller (or no) role in triangle completion. However, we hesitate to read too much into this null result. Although no significant effect of features was observed in the walking response data, Figs. 4 and 6 indicate an overall trend in favor of the distractor effect. Given the high degree of interindividual variability observed in path integration tasks (e.g., Wolbers, Wiener, Mallot, \& Buchel, 2007), it may be that we did not have enough power to show a significant effect in noisy data.

\section{General discussion}

As indicated by a ratio estimation task, walked paths associated with more features were remembered as being shorter than paths associated with fewer features. This result 
contrasts with previous results showing that path features cause an increase in remembered path length (the feature accumulation effect) and extends results from a pair of studies (Glasauer et al., 2007; Glasauer et al., 2009) in which a concurrent task caused a shortening of perceived path lengths (the distractor effect). Observation of the distractor effect in the present paradigm suggests that the effect is more general than previously indicated. The distractor effect was observed here using punctate environmental (landmark-type) distractors, vision at encoding, a filled delay between encoding and response, and a ratio estimation response mode. We consider what the extension of the distractor effect to this particular set of conditions can tell us about the underlying mechanisms.

The present demonstration of the distractor effect using punctate environmental features speaks to the relative strength of the effect. Unlike the continuous concurrent tasks used by Glasauer et al. (2007; Glasauer et al., 2009), the features used in the present study did not demand attention throughout the entire path. However, distraction from the accumulation of temporal or distance cues for even relatively small portions of a path affected remembered path length. In addition, unlike continuous concurrent tasks, punctate path features divide paths into discrete segments, which we might expect to produce an increase in remembered length (Allen, 1981; Allen \& Kirasic, 1985; JansenOsmann \& Berendt, 2005; Montello, 1997). However, any route segmentation effects that might have been at play in the present study were overpowered by the distractor effect.

Experiment 2 shows that the distractor effect is not limited to the relatively uncommon task of encoding traversed distances without vision. Glasauer and colleagues suggested that the distractor effect is caused by distraction from the accumulation of temporal cues and the consequent association of routes with shorter durations. When more perceptual distance cues are available during the encoding of walked distances (e.g., from optic flow), the relative influence of temporal information on subsequent distance estimates ought to be reduced (Montello, 2009; but see Mossio, Vida, \& Berthoz, 2008). Also, participants in this experiment could see the features coming and prepare for the new nonsense syllables, which should result in the features being easier to process and less distracting. Therefore, the results of Experiment 2 are somewhat surprising and further indicate the potential strength of the distractor effect.

Why was a feature accumulation effect seen in other, similar studies, whereas the distractor effect was observed in this study? Hutcheson and Wedell (2009) also used a concurrent task (mental arithmetic) during path encoding and a filled delay between encoding and responding. In contrast to the present study, Hutcheson and Wedell found that both of these manipulations caused an increase in the feature accumulation effect. This contrast may stem from one or more methodological differences between the studies. For example, Hutcheson and Wedell presented the paths in desktop virtual reality environments. Recall that Hutcheson and Wedell suggested that the feature accumulation effect results from relative reliance on categorical, as opposed to metric, representations. The additional idiothetic motion information sources associated with walking (e.g., proprioception, kinesthesis, efference copy) may have resulted in relatively robust metric representations in the present study (e.g., Chance, Gaunet, Beall, \& Loomis, 1998; Klatzky, Loomis, Beall, Chance, \& Golledge, 1998). The paths used in the present study were shorter on average (12.2-10.4 m vs. 12.2-24.4 m), which also may have favored metric spatial coding. Finally, the association of path features in our study with changes in the concurrent task may have made the features more distracting. Future work should continue to test which of these differences may be critical.

The present results are also striking in light of a similar study by Sadalla and Staplin (1980a) showing a feature accumulation effect. In both studies, participants' vision of the upcoming path was limited to a few feet; they followed paths marked on the floor that were approximately $6.10 \mathrm{~m}$ long and crossed by visible, linear features, and distance estimates were made using ratio estimation. For Sadalla and Staplin (1980a), features increased remembered path length; for us, features shortened remembered path length. One potentially important difference that sets the present study apart from both Sadalla and Staplin's (1980a) and Hutcheson and Wedell's (2009) is that the present experiments used both ratio estimation and triangle completion (action-based) response modes. Participants never knew, trial by trial, whether they would need to use the distance information they were encoding along the paths to support explicit symbolic responding (ratio estimation) or to navigate back to the beginning of the L path. In none of the studies showing the feature accumulation effect did participants encode path lengths with the intention of using that information to support navigation-oriented motor responses (e.g., walking).

Thus, in the present study and in those of Glasauer et al. (2007; Glasauer et al., 2009), the anticipation by participants of (potential) upcoming motor responses may have resulted in the engagement of different or additional processes during path encoding, as compared with studies showing the feature accumulation effect. For example, there is support for the idea that the anticipation of upcoming movement trajectories results in forward modeling (Philbeck, Klatzky, Behrmann, Loomis, \& Goodridge, 2001; Wolpert \& Ghahramani, 2000). Modeling movements before they occur improves spatial updating because, as the movement is executed, incoming self-motion signals can be compared against model predictions, providing an additional source of feedback. During path encoding, processes such as forward modeling that are distinct 
to situations in which specific upcoming motor responses are expected may result in the formation of spatial representations that are less susceptible to the type of heuristic, nonmetric information thought to cause feature accumulation effects. As a result, even when these representations were used to support ratio estimation responses in the present study, the distraction effect dominated. This suggests that one's intention during encoding to use distance information for action may be an important factor in determining whether the feature accumulation or the distractor effect emerges in a given situation.

If we accept Glasauer et al.'s (2007; Glasauer et al., 2009) framing of the distractor effect in terms of path integration processes and the related suggestion that the distractor effect influences kinesthetic, action-based spatial knowledge, the present results are surprising for another reason. It has been suggested that path integration and perceptual updating processes are relatively automatic (Farrell \& Robertson, 2000; Gallistel, 1990; Loomis, Klatzky, Golledge, \& Philbeck, 1999; Waller, Montello, Richardson, \& Hegarty, 2002). Survival for humans and other animals would be difficult if the ability to remain oriented with regard to immediate surroundings placed large demands on attentional resources. In the present study, distraction of attention away from spatial updating processes had a measureable effect on remembered distance (see also Takei, Grasso, Amorim, \& Berthoz, 1997; Yardley et al., 2002). Conditions in the present study (partial vision, concurrent task) may have caused what is normally an automatic process to require some attention. Future studies are needed to determine whether the distractor effect disappears under less challenging spatial updating conditions.

Finally, our discussion of the present results has been framed in terms of path features causing distraction from the accumulation of distance or temporal cues. However, we may consider explanations that do not invoke the distractor effect. Montello (2009) suggested that heuristics such as counting one's steps play a large role in how people prospectively encode walked distances, especially when perceptual information is limited. Step counting was minimized in the present study by the syllable recitation task. In addition, participants were instructed not to count syllables and not to synchronize syllables with steps, in order to discourage any explicit counting strategies. However, the concurrent task may have had an implicit influence on perceived distance. Paths without features were associated with longer periods of homogeneous syllable recitation, which may have influenced distance representations. In essence, an implicit and coarse form of syllable counting may have occurred wherein the counter was reset (to zero) at syllable changes. Future work will investigate this possibility.

In sum, the present results broaden the relevance of the distractor effect for spatial cognition. The bulk of the evidence that distraction shortens perceived extent comes from research into temporal processing (e.g., Brown, 1997). Therefore, the present demonstration of the distractor effect in the spatial realm supports suggestions that temporal and spatial processing share a common cognitive and neural architecture.

Acknowledgments Thanks to Owen Footer and Jacob Marks for invaluable help collecting and analyzing these data. This research was supported by NIH grants R01 AG031150, principle investigator Jeffrey Zacks, and R01 EY021771, principle investigator John Philbeck.

\section{References}

Allen, G. L. (1981). A developmental perspective on the effects of "subdividing" macrospatial experience. Journal of Experimental Psychology: Human Learning and Memory, 7, 120-132.

Allen, G. L., \& Kirasic, K. C. (1985). Effects of the cognitive organization of route knowledge on judgments of macrospatial distance. Memory \& Cognition, 13, 218-227.

Berthoz, A., Israel, I., Georges-Francois, P., Grasso, R., \& Tsuzuku, T. (1995). Spatial memory of body linear displacement: What is being stored? Science, 269(5220), 95-98.

Briggs, R. (1973). Urban cognitive distance. In R. Downs \& D. Stea (Eds.), Image and environment: Cognitive mapping and spatial behavior (pp. 361-388). New Brunswick, NJ: Aldine.

Brooks, L. R. (1968). Spatial and verbal components of the act of recall. Canadian Journal of Psychology/Canadian Review of Psychology, 22(5), 349-368.

Brown, S. W. (1997). Attentional resources in timing: Interference effects in concurrent temporal and nontemporal working memory tasks. Perception \& Psychophysics, 59(7), 1118-1140.

Brown, S. W., \& West, A. N. (1990). Multiple timing and the allocation of attention. Acta Psychologica, 75(2), 103-121.

Chance, S. S., Gaunet, F., Beall, A. C., \& Loomis, J. M. (1998). Locomotion mode affects the updating of objects encountered during travel: The contribution of vestibular and proprioceptive inputs to path integration. Presence, 7(2), 168-178.

Farrell, M. J., \& Robertson, I. H. (2000). The automatic updating of egocentric spatial relationships and its impairment due to right posterior cortical lesions. Neuropsychologia, 38, 585-595.

Fujita, N., Klatzky, R. L., Loomis, J. M., \& Golledge, R. G. (1993). The encoding-error model of pathway completion without vision. Geographical Analysis, 25(4), 295-314.

Gallistel, C. R. (1990). The organization of learning. Cambridge, MA: MIT Press.

Glasauer, S., Schneider, E., Grasso, R., \& Ivanenko, Y. P. (2007). Space-time relativity in self-motion reproduction. Journal of Neurophysiology, 97(1), 451-461.

Glasauer, S., Stein, A., Gunther, A. L., Flanagin, V. L., Jahn, K., \& Brandt, T. (2009). The effect of dual tasks in locomotor path integration. Annals of the New York Academy of Sciences, 1164(1), 201-205.

Gobet, F., Lane, P. C. R., Croker, S., Cheng, P. C. H., Jones, G., Olivier, I., \& Pine, J. M. (2001). Chunking memories in human learning. Trends in Cognitive Science, 5, 236-243.

Hicks, R. E., Miller, G. W., Gaes, G., \& Bierman, K. (1977). Concurrent processing demands and the experience of time-in-passing. The American Journal of Psychology, 90(3), 431-446.

Holyoak, K. J., \& Mah, W. A. (1982). Cognitive reference points in judgments of symbolic magnitudes. Cognitive Psychology, 14, $328-352$. 
Hutcheson, A. T., \& Wedell, D. H. (2009). Moderating the route angularity effect in a virtual environment: Support for a dual memory representation. Memory \& Cognition, 37(4), 514-521.

Huttenlocher, J., Hedges, L. V., \& Duncan, S. (1991). Categories and particulars: Prototype effects in estimating spatial location. Psychological Review, 98(3), 352-376.

Jansen-Osmann, P., \& Berendt, B. (2002). Investigating distance knowledge using virtual environments. Environment and Behavior, 34(2), 178-193.

Jansen-Osmann, P., \& Berendt, B. (2005). What makes a route appear longer? An experimental perspective on features, route segmentation and distance knowledge. The Quarterly Journal of Experimental Psychology, 58A(8), 1390-1414.

Jansen-Osmann, P., \& Wiedenbauer, G. (2006). Distance cognition in virtual environmental space: Further investigations to clarify the route-angularity effect. Psychological Research (Psychologische Forschung), 70(1), 43-51.

Klatzky, R. L., Beall, A. C., Loomis, J. M., Golledge, R. G., \& Philbeck, J. W. (1999). Human navigation ability: Tests of the encoding-error model of path integration. Spatial Cognition and Computation, 1(1), 31-65.

Klatzky, R. L., Loomis, J. M., Beall, A. C., Chance, S. S., \& Golledge, R. G. (1998). Spatial updating of self-position and orientation during real, imagined, and virtual locomotion. Physiological Science, 9(4), 293-298.

Loomis, J. M., Klatzky, R. L., Golledge, R. G., Cicinelli, J. G., Pellegrino, J. W., \& Fry, P. (1993). Nonvisual navigation by blind and sighted: Assessment of path integration ability. Journal of Experimental Psychology. General, 122(1), 73-91.

Loomis, J. M., Klatzky, R. L., Golledge, R. G., \& Philbeck, J. W. (1999). Human navigation by path integration. In R. G. Golledge (Ed.), Wayfinding behavior: Cognitive mapping and other spatial processes (pp. 125-151). Baltimore: Johns Hopkins UP.

McNamara, T. P., Hardy, J. K., \& Hirtle, S. C. (1989). Subjective hierarchies in spatial memory. Journal of Experimental Psychology: Learning, Memory, and Cognition, 15(2), 211-227.

Montello, D. R. (1997). The perception and cognition of environmental distance: Direct sources of information. In S. C. Hirtle \& A. U. Frank (Eds.), Spatial information theory: A theoretical basis for GIS (pp. 297-311). Berlin: Springer-Verlag, Lecture Notes in Computer Science 1329.

Montello, D. R. (2009). A conceptual model of the cognitive processing of environmental distance information. In K. S. Hornsby, C. Claramunt, M. Denis, \& G. Ligozat (Eds.), Spatial information theory (pp. 1-17). Proceedings of COSIT '09. Berlin, Heidelberg: Springer-Verlag, Lecture Notes in Computer Science 5756.
Mossio, M., Vidal, M., \& Berthoz, A. (2008). Traveled distances: New insights into the role of optic flow. Vision Research, 48(2), 289-303.

Philbeck, J. W., Klatzky, R. K., Behrmann, M., Loomis, J. M., \& Goodridge, J. (2001). Active control facilitates non-visual navigation. Journal of Experimental Psychology: Human Perception and Performance, 27, 141-153.

Sadalla, E. K., \& Magel, S. G. (1980). The perception of traversed distance. Environment and Behavior, 12(1), 65-79.

Sadalla, E. K., \& Staplin, L. J. (1980a). The perception of traversed distance: Intersections. Environment and Behavior, 12(2), 167-182.

Sadalla, E. K., \& Staplin, L. J. (1980b). An information storage model for distance cognition. Environment and Behavior, 12(2), 183-193.

Sadalla, E. K., Staplin, L. J., \& Burroughs, W. J. (1979). Retrieval processes in distance cognition. Memory \& Cognition, 7(4), 291296.

Sargent, J., Dopkins, S., Philbeck, J., \& Chichka, D. (2010). Chunking in spatial memory. Journal of Experimental Psychology: Learning, Memory, and Cognition, 36(3), 576-589.

Takei, Y., Grasso, R., Amorim, M.-A., \& Berthoz, A. (1997). Circular trajectory formation during blind locomotion: A test for path integration and motor memory. Experimental Brain Research, $115(2), 361-368$.

Waller, D., Montello, D. R., Richardson, A. E., \& Hegarty, M. (2002). Orientation specificity and spatial updating of memories for layouts. Journal of Experimental Psychology: Learning, Memory, and Cognition, 28(6), 1051-1063.

Wang, R. F., \& Brockmole, J. R. (2003). Simultaneous spatial updating in nested environments. Psychonomic Bulletin \& Review, 10, 981-986.

Wolbers, T., Wiener, J. M., Mallot, H. A., \& Buchel, C. (2007). Differential recruitment of the hippocampus, medial prefrontal cortex, and the human motion complex during path integration in humans. The Journal of Neuroscience, 27(35), 9408-9416.

Wolpert, D. M., \& Ghahramani, Z. (2000). Computational principles of movement neuroscience. Nature Neuroscience, 3, 1212-1217.

Yardley, L., Papo, D., Bronstein, A., Gresty, M., Gardner, M., Lavie, N., \& Luxon, L. (2002). Attentional demands of continuously monitoring orientation using vestibular information. Neuropsychologia, 40(4), 373-383.

Zacks, J. M., Speer, N. K., Swallow, K. M., Braver, T. S., \& Reynolds, J. R. (2007). Event perception: A mind-brain perspective. Psychological Bulletin, 133(2), 273-293.

Zakay, D. (1989). Subjective time and attentional resource allocation: An integrated model of time estimation. In I. Levin \& D. Zakay (Eds.), Time and Human Cognition (pp. 365-397). Amsterdam: North-Holland. 